\title{
ST Changes Observed in Short Spaced Bipolar Leads Suitable for Patch Based Monitoring
}

\author{
Michael Jennings ${ }^{1}$, Daniel Guldenring ${ }^{2}$, Raymond Bond ${ }^{1}$, Ali Rababah ${ }^{1}$, James McLaughlin ${ }^{1}$, Dewar \\ D Finlay $^{1}$ \\ ${ }^{1}$ Ulster University, Newtownabbey, UK \\ ${ }^{2}$ HTW Berlin, Berlin, Germany
}

\begin{abstract}
This article investigates the selection of optimal ECG leads for the detection of ST changes more likely to appear in patch systems with closely spaced leads. Method: We analysed body surface potential maps (BSPMs) from 44 subjects undergoing PTCA. BSPMs were recorded at 120 sites and these were expanded to 352 nodes (Dalhousie torso) using Laplacian interpolation. A total of 88 BSPMs were investigated. This included the 44 subjects at baseline and the 44 subjects at peak balloon inflation (PBI). At PBI the subjects had various coronary arteries occluded (14 LAD, 15 LCX, 15 RCA). All possible bipolar leads were calculated for each subject. Leads were ranked based on the maximum ST-segment change between baseline and PBI for each subject. Leads with electrode spacing of more than $100 \mathrm{~mm}$ were excluded. The highest ranked lead was chosen as the short spaced lead (SSL) on the anterior torso. Result: The median ST-segment change for the chosen SSL for each vessel was $L A D=134 \mu V, L C X$ $=65 \mu V, R C A=166 \mu V$. The maximum $S T$ segment change observed for the same lead was $L A D=277 \mu V, L C X=166$ $\mu V, R C A=257 \mu V$. For comparison, the highest median observed on the 12-lead ECG for each vessel was $L A D=$ $137 \mu V(V 3), L C X=130 \mu V(I I I), R C A=196 \mu V(I I I)$.
\end{abstract}

\section{Introduction}

The 12-lead ECG is still the most important tool for detecting acute coronary syndromes, with consideration of at least two contiguous leads required for diagnosis [1]. However, the development of new patch-based short spaced lead (SSL) ECG systems to detect cardiac defects has increased dramatically, with a lack of academic literature investigating their performance [2]. Novel techniques of ambulatory monitoring are capable of storing and transmitting ECG data for diagnostic purposes [3]. Patch devices have shown accurate cardiac arrhythmia detection, however, a single lead is limited in the localisation of di- agnoses compared to a 12-lead ECG [4]. Deviation from 12-lead ECG configurations has been shown to reduce the diagnostic accuracy of algorithms relying on current ST amplitude criteria $[5,6]$. The $\mathrm{Zio}^{\circledR} \mathrm{XT}$ is an example of an existing SSL patch-based ECG monitor designed to challenge existing diagnostic methods, particularly with longer recording duration and automated arrhythmia detection [7]. Many patch-based ECG devices are designed to detect specific arrhythmia based on torso placement with a lack of specificity to ST-segment changes [7]. Further studies have discussed novel vessel-specific leads (VSLs) to detect ST-elevation myocardial infarction (STEMI) [8]. We aim to investigate a new short-spaced lead (SSL) as a means to detect ST-elevation (STE) constrained to $100 \mathrm{~mm}$ between electrodes suitable for continuous monitoring.

\section{Method}

The proposed method uses body surface potential maps (BSPM) to investigate lead placement in the detection of STE for short spaced leads (SSL).

\subsection{Data}

The data are previously described in $[9,10]$ and include recordings from 44 subjects undergoing elective percutaneous transluminal coronary angioplasty (PTCA). Data were recorded, with respect to the Wilson central terminal, at 120 leads. After recording, these 120 leads were further expanded to potentials at the locations of 352 nodes through Laplacian interpolation. All subjects had single-vessel coronary heart disease and had positive ECG changes upon balloon inflation in one-of-three coronary arteries: left anterior descending (LAD, $\mathrm{n}=14)$, left circumflex (LCX, n=15) and the right coronary artery (RCA, $\mathrm{n}=15$ ). Data were available at baseline (absence of STelevation) and during PBI. ECGs during PBI were assumed to represent changes compatible with those observed in patients suffering ischaemic episodes. 


\subsection{Algorithm}

MATLAB 2018b was used to calculate all possible lead combinations $(n=123904)$ from all possible pairs of the 352 nodes. The signal amplitude was extracted from $40 \mathrm{~ms}$ after the $\mathrm{J}$-point $(\mathrm{J}+40 \mathrm{~ms})$ to give a representative measure of ST-segment value. The difference at $\mathrm{J}+40 \mathrm{~ms}$ between baseline and PBI was calculated. This process was repeated for all leads created previously. Each lead was ranked in descending order of absolute ST-segment change $(\Delta \mathrm{ST})$. This process was repeated for each subject $(n=44)$, where the rank given to a lead for the previous subject is added to the rank of the next subject. The lowest ranked value denotes the lead with the highest $\Delta \mathrm{ST}$ across all subjects. A generic 3D torso (Dalhousie torso) described in a previous study [9] was used to calculate the distance between electrodes for each lead. Leads with electrode spacing greater than $100 \mathrm{~mm}$ apart were excluded. The SSL was selected as the lowest ranked lead from remaining leads $(n=9760)$. Figure 1 shows this process.

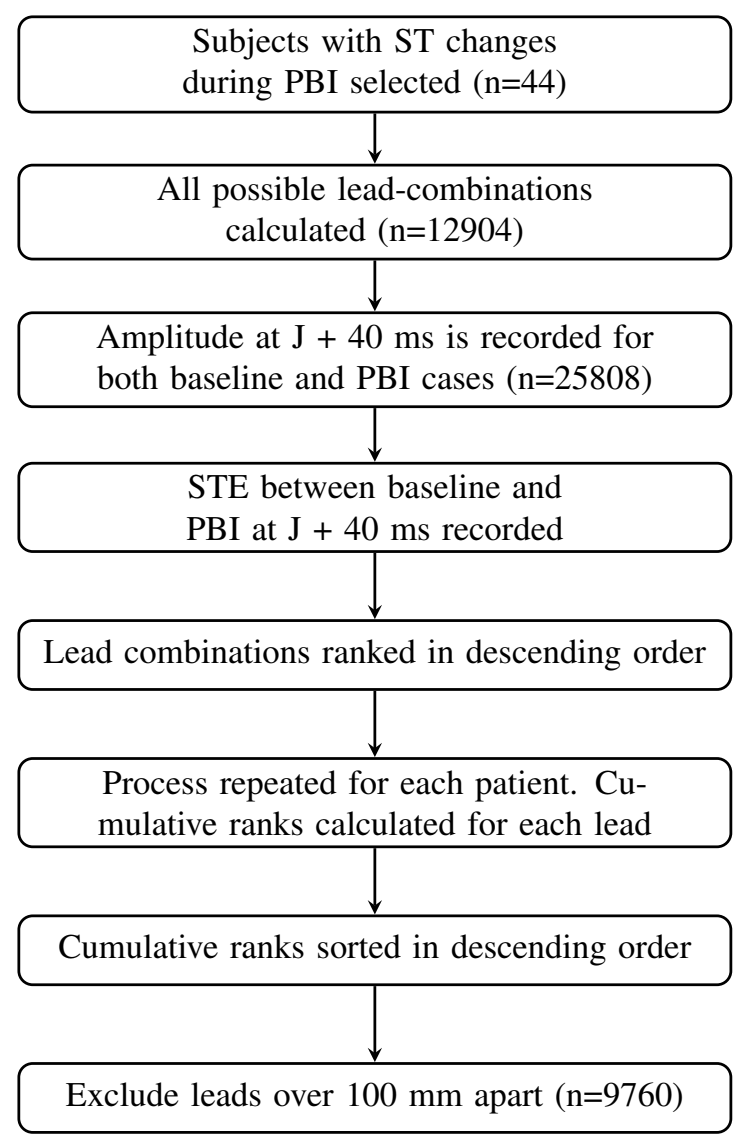

Figure 1. Sorting algorithm

\section{Results}

\subsection{Position of selected lead}

The selected SSL which reflected the highest STsegment change within the physical $100 \mathrm{~mm}$ constraint was identified as being on the anterior torso between a region in the left precordium and a more inferior abdominal region. Specifically, according to the node numbering on the Dalhousie torso, the SSL was positioned between an electrode superior to V3 (Dalhousie torso node 173) and an electrode left of the sagittal axis between the epigastric and umbilical abdominal regions (Dalhousie torso node 254). Figure 2 illustrates the position of the SSL with respect to the the six precordial leads of the 12-lead ECG. The BSPM shows the median observed signal amplitude during PBI at $\mathrm{J}+40 \mathrm{~ms}$ for subjects undergoing LAD occlusion PTCA $(n=14)$.

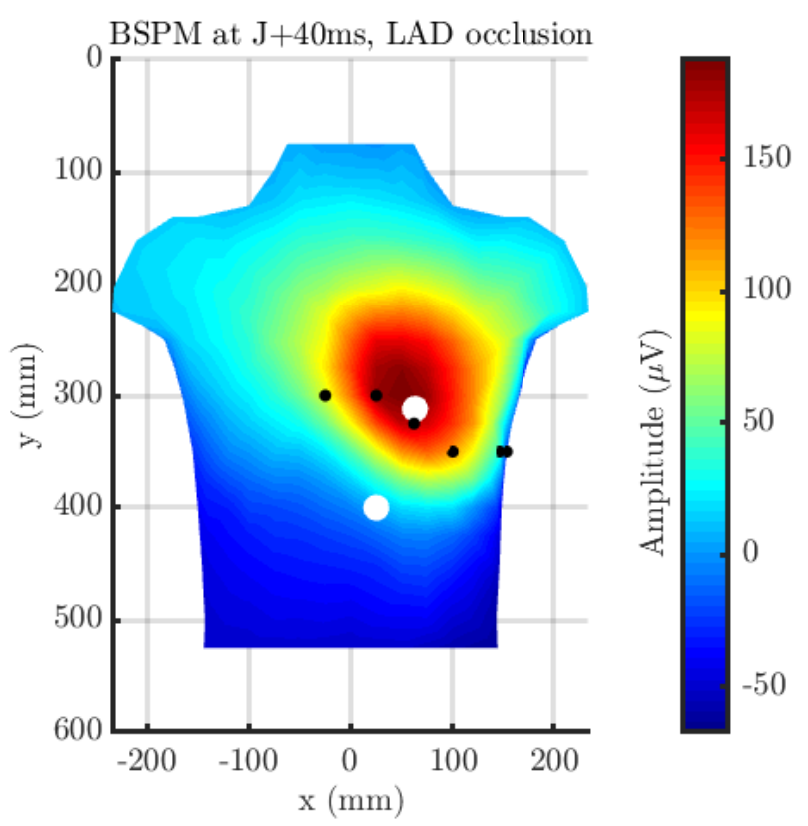

Figure 2. Anterior torso median-amplitude BSPM of subjects at J+40 ms during LAD occlusion PTCA $(n=14)$. White $=$ SSL; black $=$ V-leads.

\subsection{ST segment changes on selected lead}

Figure 3 shows the absolute change in ST-elevation $(\Delta \mathrm{ST})$ across all subjects as median, 25th and 75th percentiles. The SSL has a median $\Delta \mathrm{ST}$ of $125 \mu \mathrm{V}$ with a maximum value of $277 \mu \mathrm{V}$. This performs comparatively with the precordial lead V2. VSLs from a previous study [8] are used in comparison with the SSL and 12-lead ECG for a critical analysis. The median $\triangle \mathrm{ST}$ for VSLs are as follows: $\mathrm{LAD}=156 \mu \mathrm{V}, \mathrm{LCX}=162 \mu \mathrm{V}, \mathrm{RCA}=187 \mu \mathrm{V}$. 


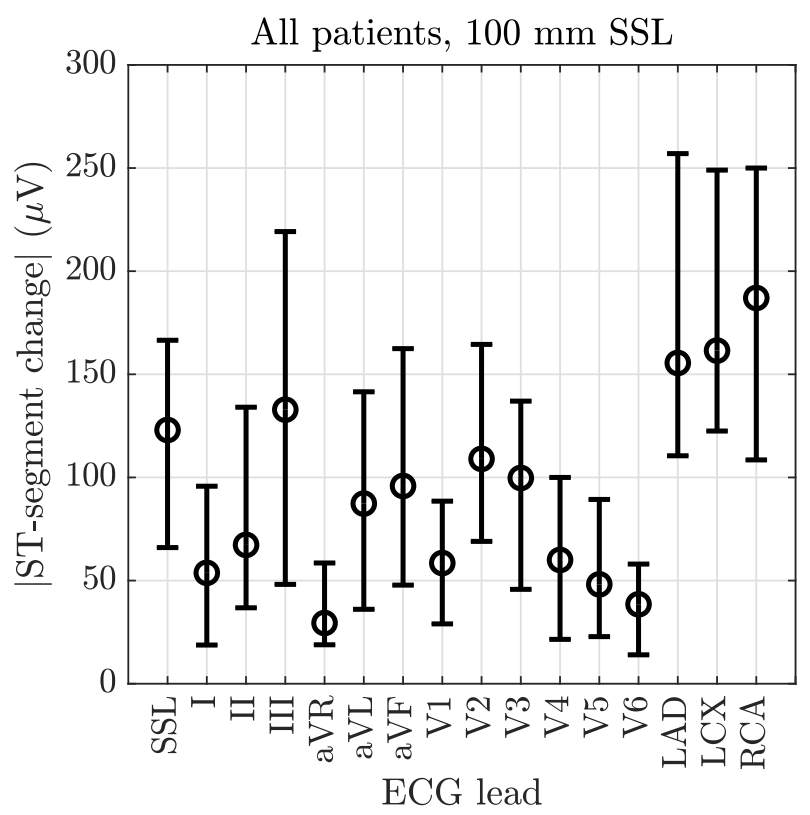

Figure 3. $\Delta$ ST across all leads, all subjects $(n=44)$

\subsection{LAD occlusion}

To further analyse the SSL performance, it is necessary to look at specific vessel occlusions. In this example, only subjects with LAD occlusion are considered with the same method as described in Section $2.2(n=14)$. We observe a median $\Delta \mathrm{ST}$ of $134 \mu \mathrm{V}$ in the SSL, comparable with the precordial leads $\mathrm{V} 2$ and $\mathrm{V} 3$ both showing $137 \mu \mathrm{V} \Delta \mathrm{ST}$. This is $36 \%$ lower than the relevant VSL. The maximum SSL $\Delta$ ST recorded across all subjects was during LAD occlusion at $277 \mu \mathrm{V}$. Figure 4 shows the performance of each lead at $\mathrm{J}+40 \mathrm{~ms}$ across LAD PTCA subjects.

\subsection{LCX occlusion}

In subjects undergoing PTCA in the LCX coronary artery $(n=15)$, we observe a $\Delta \mathrm{ST}$ median of $65 \mu \mathrm{V}$ in the SSL. The SSL performs comparatively to V3, with a median of $58 \mu \mathrm{V}$. Figure 5 illustrates the SSL characteristics. This is the lowest $\Delta \mathrm{ST}$ value observed across the three coronary arteries at $63 \%$ below the VSL. The maximum $\Delta \mathrm{ST}$ in the SSL was $166 \mu \mathrm{V}$.

\subsection{RCA occlusion}

$\triangle \mathrm{ST}$ observed in RCA PTCA subjects $(\mathrm{n}=15)$ possess the highest overall values. The SSL shows a median $\Delta \mathrm{ST}$ of $166 \mu \mathrm{V}, 28 \%$ below the relevant VSL. The SSL exhibits similar ST-segment changes to aVF which has a median $\Delta \mathrm{ST}$ of $151 \mu \mathrm{V}$ across subjects. Figure 6 shows the SSL performance. The maximum $\Delta \mathrm{ST}$ in the SSL was $257 \mu \mathrm{V}$.

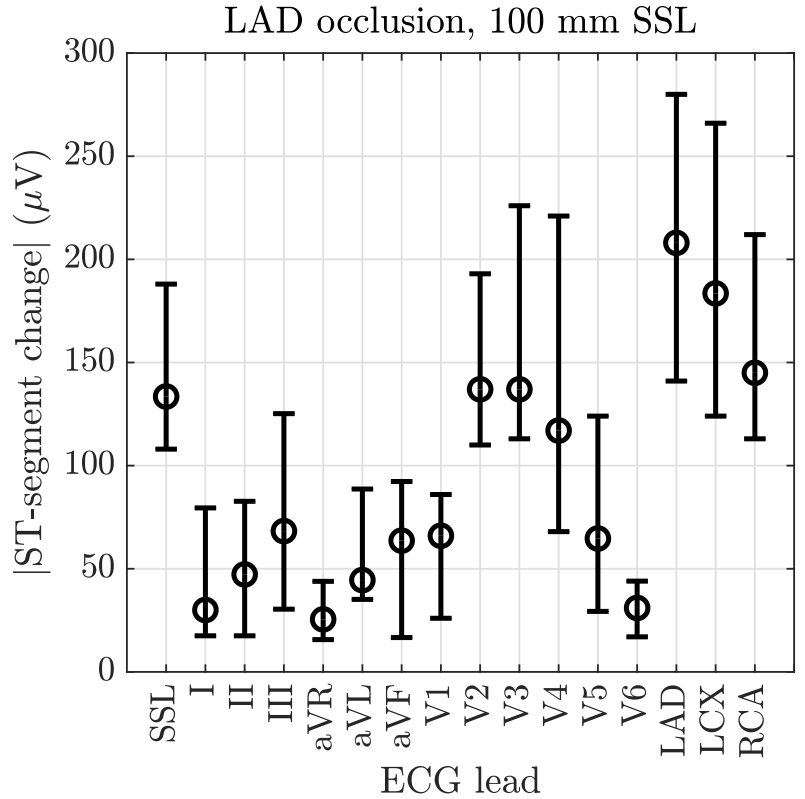

Figure 4. $\Delta \mathrm{ST}$ deviation, LAD occlusions only $(\mathrm{n}=14)$

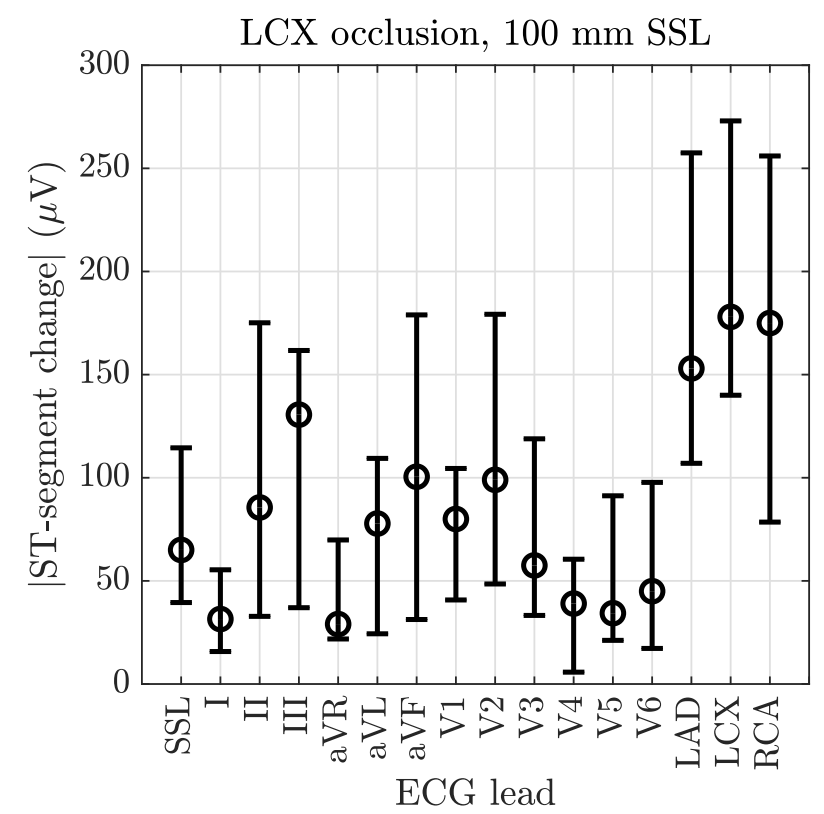

Figure 5. $\Delta$ ST deviation, LCX occlusions only $(\mathrm{n}=15)$ 


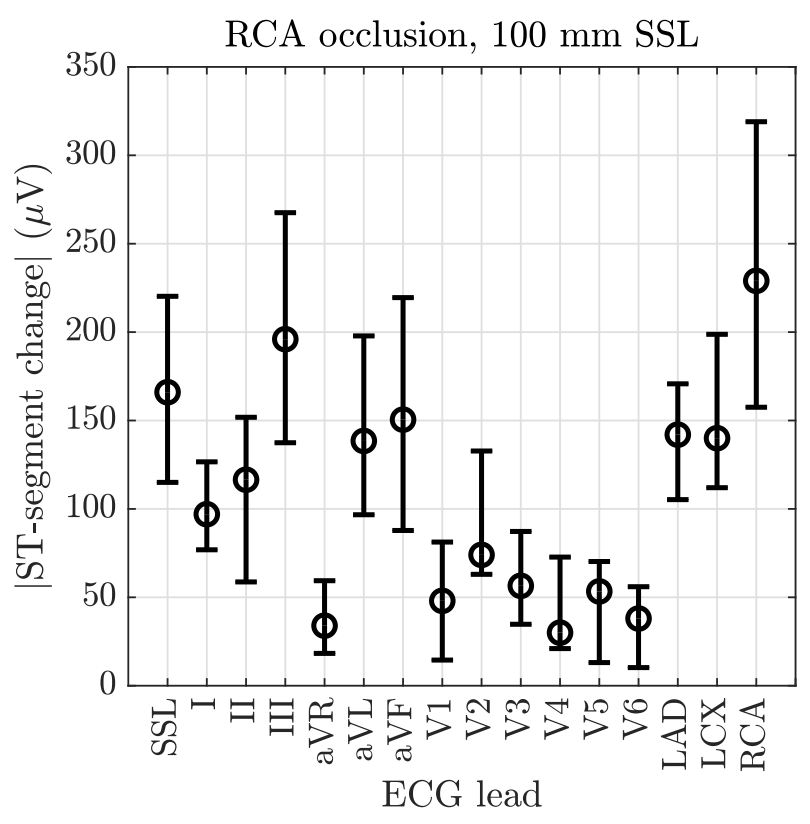

Figure 6. $\Delta$ ST deviation, RCA occlusions only $(n=15)$

\section{Conclusion}

Our analysis has shown the identified SSL performs comparatively with the existing 12-lead setup for detecting ST-segment changes. The SSL shows the highest performance during RCA occlusion which has been verified by the associated body surface potential maps and previously studied vessel specific leads. Although the findings of this study support an SSL-based method of detecting ST elevation, a larger dataset is required with more complex coronary artery lesions to verify the results. Patient specific 3D torso models would improve the location accuracy of the chosen lead and account for anatomical variability. Furthermore, the need for at least two contiguous leads for STEMI detection reduces the impact of an SSL for clinical use. Specifically, these studies may investigate the use of SSLs toward detection of MI and their use in patch-based ECG.

\section{Acknowledgements}

This project is part of the Eastern Corridor Medical Engineering centre (ECME). It is supported by the European Union's INTERREG VA Programme, managed by the Special EU Programmes Body (SEUPB).

\section{References}

[1] Thygesen K, Alpert JS, Jaffe AS, Chaitman BR, Bax JJ, Morrow DA, White HD, et al. Fourth universal definition of myocardial infarction (2018). Journal of the American College of Cardiology 2018;72(18):2231-2264.

[2] Bansal A, Joshi R. Portable out-of-hospital electrocardiography: A review of current technologies. Journal of Arrhythmia 2018;34(2):129-138.

[3] Hasan MK, Shahjalal M, Chowdhury MZ, Jang YM. Realtime healthcare data transmission for remote patient monitoring in patch-based hybrid occ/ble networks. Sensors Basel Switzerland 2019;19(5).

[4] Karaoğuz MR, Yurtseven E, Aslan G, Deliormanlı BG, Adıgüzel Ö, Gönen M, Li KM, Yılmaz EN. The quality of ecg data acquisition, and diagnostic performance of a novel adhesive patch for ambulatory cardiac rhythm monitoring in arrhythmia detection. Journal of electrocardiology 2019; 54:28-35.

[5] Bond R, Finlay DD, Guldenring D, Breen C. Data driven computer simulation to analyse an ecg limb lead system used in connected health environments. Methods of information in medicine 2016;55(03):258-265.

[6] Guldenring D, Finlay DD, Bond RR, Kennedy A, McLaughlin J. Limitations of the smartphone based single ECG-lead evaluation of STEMI. Journal of Electrocardiology jan 2018;51(1):e3.

[7] Fung E, Järvelin MR, Doshi RN, Shinbane JS, Carlson SK, Grazette LP, Chang PM, Sangha RS, Huikuri HV, Peters NS. Electrocardiographic patch devices and contemporary wireless cardiac monitoring. Frontiers in physiology 2015; 6:149.

[8] Horáček BM, Mirmoghisi M, Warren JW, Wagner GS, Wang JJ. Detection of myocardial ischemia by vesselspecific leads derived from the 12-lead electrocardiogram and its subsets. Journal of electrocardiology 2008; 41(6):508-517.

[9] Horáček BM, Clements JC. The inverse problem of electrocardiography: A solution in terms of single-and doublelayer sources on the epicardial surface. Mathematical biosciences 1997;144(2):119-154.

[10] Dawoud F. Using inverse electrocardiography to image myocardial infarction. In 2007 Computers in Cardiology. IEEE, 2007; 177-180.

Address for correspondence:

Michael Jennings

NIBEC, Ulster University

NEWTOWNABBEY, United Kingdom

jennings-m5@ulster.ac.uk 